\title{
The Lost Banlieues of the Republic? Introduction
}

\author{
Frédéric Viguier \\ New York University
}

Que se passe-t-il dans les banlieues populaires françaises ? Sur le constat, il ne se trouve guère de voix dissonante : les grands ensembles de la périphérie des villes françaises sont affligés de l'ensemble des maux de la société française. Les lieux sont laids et inhumains et furent bâtis trop vite dans les années 1960 et 1970 ; ils sont également mal reliés aux centres urbains ou aux espaces plus dynamiques économiquement. Un temps tentées par la modernité de ces nouveaux quartiers, les classes moyennes les ont fuis depuis longtemps. Quant aux franges supérieures des classes populaires, elles s'efforcent de suivre cet exode ; ceux qui n'en ont pas les moyens en conçoivent une souffrance immense. Les populations immigrées que le chômage massif empêche désormais d'intégrer y sont majoritaires ; les jeunes désoeuvrés y sont sans perspectives d'avenir.

En revanche, sur l'analyse des causes ou des remèdes à apporter, les avis divergent : entre gauche et droite, entre gauche de gouvernement et gauche "de gauche », entre presse écrite et presse de télévision, entre savants et experts, etc. Ces débats ont d'autant plus de vigueur que les principaux concernés n'ont pas la possibilité de parler d'eux-mêmes sinon par fragments à l'occasion de tel ou tel entretien collectif dont les reportages télévisés sont friands. Dans ces controverses, les sciences sociales aiment se représenter leurs analyses comme opposées aux simplifications sensationnelles de la vision «médiatico-politique » mais échouent souvent à faire entendre leur voix tant est grande la demande de solutions et de réponses énergiques et tant cette impatience porte à disqualifier les « excuses sociologiques ». Peut-être aussi peinent-elles à convaincre par manque d'enquêtes ethnographiques minutieuses. Il peut paraître naïf d'invoquer le travail de terrain quand tout le monde s'en réclame, mais cet objectif doit demeurer celui des sciences sociales 
qui travaillent sur les problèmes sociaux en banlieue, afin de pouvoir restituer l'espace des points de vue et la logique de production des comportements.

On sait bien compter les injustices-chômage ou discriminations ethniques-mais si ces chiffres frappants excusent, ils n'expliquent pas les enchaînements, l'irruption d'explosions. Rares sont en effet les travaux qui inscrivent les problèmes des banlieues dans l'histoire récente du monde populaire (ouvrier et immigré), qui savent faire sentir l'augmentation de la violence vécue subjectivement par le voisinage, qui permettent de comprendre le déroulement des carrières violentes, l'apprentissage de la culture de provocation dans les espaces publics, le récit vécu d'une émeute, les rapports entre générations et entre sexes. Il y a à cette absence de bonnes raisons : le temps long d'une ethnographie dans un milieu difficile, méfiant sinon hostile, se prête mal aux contraintes de productivité de la vie académique, et l'inconfort d'une situation d'enquête dans un grand ensemble "sensible » requiert, pour le surmonter ou le minimiser, des propriétés sociales rares chez les doctorants ou chercheurs en sciences sociales.

De ce fait, l'explication qui prédomine dans l'espace public consiste à accuser la volonté sécessionniste de certaines populations par rapport à cette forme française particulière de l'État-nation démocratique qu'est la République. Telle est en effet la formule explicative : l'engrenage du chômage et de la ségrégation ethnique favoriseraient le goût pour l'entre-soi communautaire et conduiraient au refus violent de la société française que les émeutes du mois de novembre 2005 n'ont fait que confirmer de manière spectaculaire. Depuis longtemps déjà, les enseignants de banlieue auraient constaté le repli communautaire attesté dans les collèges et les lycées de la République par le refus d'apprendre Voltaire et Rousseau au nom du Coran. Des jeunes de plus en plus jeunes y commettraient des violences de plus en plus grandes. Y règneraient en maîtres des trafics de toutes sortes et ces démons nouveaux que sont le fondamentalisme religieux, l'antisémitisme, et le sexisme, démons que l'on croyait devenus impossibles en terre laïque, après la Shoah et à l'heure de l'émancipation des femmes. Bref, le temps paraît loin où la banlieue connotait les plaisirs des parties de campagne et des bals musette au bord de la Marne ; elle n'évoque plus les beaux quartiers de l'ouest parisien et ne crée pas non plus les inquiétudes ou les espoirs que suscitaient les banlieues rouges ouvrières. Désormais c'est la condamnation qui prévaut, qu'elle porte sur le système, version euphémisée, ou sur ses jeunes résidents. L'explication appelle dans les deux cas une "sérieuse reprise en main », dont les efforts porteraient d'abord sur les habitants. Serait en jeu un "délitement culturel et politique de la nation ", comme l'affirmait la conclusion d'un ouvrage largement médiatisé en 2002, consacré au milieu scolaire de la région parisienne et intitulé Les Territoires perdus de la République ${ }^{1}$.

Répétons à nouveau qu'il ne s'agit nullement ici de révoquer ces diagnostics pour les qualifier de purs fantasmes. En revanche, la compréhension à la fois territoriale (les banlieues) et nationale (la République) du problème social 
nous paraît pouvoir être questionnée. C'est pourquoi ce numéro spécial paraphrase ce titre en lui adjoignant un point d'interrogation : Les banlieues perdues de la République ? Les articles présentés, tout comme les quatre interventions du colloque dont ils sont issus ${ }^{2}$, s'efforcent de reconstruire la logique des différents producteurs de diagnostics et de traitement du problème social des banlieues. Il ne s'agit pas d'un vain détour loin du « vrai » problème : les souffrances et les réactions des « jeunes des cités ». C'est un réflexe sociologique essentiel que d'examiner avec soin l'histoire sociale de la construction d'un problème social. Surtout, ces enquêtes nous mettent également en contact avec des personnes réelles, forcées de cohabiter souvent dans le conflit et la douloureuse incompatibilité avec des gens qu'elles ne peuvent plus toujours comprendre : que ce soit le personnel communiste municipal (texte d'Olivier Masclet), les travailleurs sociaux (Sylvie Tissot), ou les enseignants (Frédéric Viguier).

Ne s'agit-il vraiment que des banlieues ? Telle est l'interrogation initiale de l'enquête de Sylvie Tissot. Son travail analyse la genèse de cette conception territoriale des problèmes sociaux et la rapporte à la mise en place de la "Politique de la ville ", où se reconvertissent les chargés d'études précaires, laissés sans emploi par la contraction de l'activité planificatrice produit de la contraction du marché des études et sans cause par la réduction des possibilités militantes. «Banlieues perdues pour qui ? »demande d'une certaine façon le travail d'Olivier Masclet : pour ses habitants orphelins d'un mouvement ouvrier défait. Par qui ? Par le communisme municipal, mauvais stratège peutêtre, mais assurément désemparé par les processus de différenciation sociale que la crise économique a engendrés dans les anciennes banlieues rouges. Dans ce silence politique, une voix porte fort : celle des enseignants dont l'ethos méritocratique est fortement ébranlé par les transformations du système éducatif et la dévalorisation spectaculaire des titres scolaires. L'article de Frédéric Viguier montre comment les ressources sont faibles pour faire face aux contradictions de leur nouveau rôle. Ils sont alors souvent conduits à considérer l'enseignement dispensé comme un bien de salut culturel plutôt que comme une clé pour le monde professionnel et, finalement, à se préoccuper d'ajustement culturel plus que d'égalisation des chances. Quant aux " grands » intellectuels, ils sont également menacés par le déclin de l'institution scolaire et se montrent nostalgiques du monde républicain d'hier. Pourtant cette République aujourd'hui idéalisée fut également coloniale. Nacira Guénif-Souilamas repère dans les figures épouvantables du « garçon arabe » et de la « fille voilée » d'aujourd'hui les avatars post-coloniaux sexués des anciens indigènes de la République. 


\section{Notes}

1. Emmanuel Brenner, dir., Les Territoires perdus de la République. Antisémitisme, racisme et sexisme en milieu scolaire (Paris : Fayard/Mille et une nuits, 2002).

2. Colloque "The Lost Banlieues of the Republic », Institute of French Studies, New York University, New York, 5-6 novembre 2004. 